\begin{tabular}{|c|c|}
\hline Title & Numerical reconstruction of an infrared wavefront utilizing an optical phase modulation device \\
\hline Author(s) & Miyamoto, Naoki; Nisiyama, Shusuke; Tomioka, Satoshi; Enoto, Takeaki \\
\hline Citation & $\begin{array}{l}\text { Optics Communications, 272(1), 67-72 } \\
\text { https://doi.org/10.1016/.optcom.2006.11.018 }\end{array}$ \\
\hline Issue Date & 2007-04-01 \\
\hline Doc URL & http:/hdl.handle.net/2115/20442 \\
\hline Type & article (author version) \\
\hline File Information & naoki_miyamoto_v2.pdf \\
\hline
\end{tabular}

Instructions for use 


\title{
Numerical reconstruction of an infrared wavefront utilizing an optical phase modulation device
}

\author{
Naoki Miyamoto*, Shusuke Nisiyama, Satoshi Tomioka, \\ Takeaki Enoto \\ Graduate School of Engineering, Hokkaido University, Sapporo 060-8628, Japan
}

\begin{abstract}
We utilize nitroanisole, that absorbs infrared(IR) radiation as heat, as an optical modulation device based on a thermal process. The nitroanisole exhibits a thermal lens effect, i.e. a temperature dependent refractive index. Hence, the nitroanisole can induce phase modulation to visible light, in direct response to intensity of the incident IR radiation. The proposed method can be used to obtain the phase modulation distribution that corresponds to the IR intensity distribution, i.e. the IR hologram itself, on the nitroanisole by examining the phase map of visible light that is modulated upon passing through the nitroanisole. The IR wavefront can be reconstructed by calculating extracted IR holograms through the Fresnel transform. It is verified that both the amplitude and the phase of the IR wavefront can be reconstructed accurately by proposed method.
\end{abstract}

Key words: Digital holography; Infrared holography; Nitroanisole; Phase modulation

Preprint submitted to Elsevier Science

7 December 2006 


\section{Introduction}

With the development of the high resolution charge-coupled device(CCD), digital holography techniques[1-3] that uses a CCD as a holographic recording medium may now be applied practically for 3D-image reconstruction, surface profile measurement, etc. This technique is able to attain high accuracy by combining phase shifting methods. A visible light source is mainly used for digital holography since conventional CCDs are designed to be sensitive to visible light. If this technique can be used in middle and far infrared(IR) regions in the same way, its breadth of applications can be expanded to various fields, such as analysis of large defects or deformations, medical diagnosis, nondestructive inspection, etc. Regarding for holographic recording media for use in the IR spectrum region, detectors, such as pyroelectric devices, $\mathrm{HgCdTe}$ diodes, etc, which are sensitive to IR radiation can record the IR holograms[4]. On the other hand, the use of some holographic recording materials based on a thermal process at the wavelength of $10.6 \mu \mathrm{m}$ has been also studied [58]. Although the time resolution or the sensitivity of such materials is lower than that of opto-electric detectors, they have sufficiently spatial resolution to record the IR holograms. An IR wavefront can be reconstructed easily with illuminating these materials(IR holograms) by a visible laser. However phase maps of the IR wavefront are not obtained in this method since only intensity distribution of reconstructed images is observed.

In order to reconstruct not only amplitude but also phase of the IR wavefront, we propose a method to digitally extract IR holograms by utilizing nitroanisole

\footnotetext{
* Corresponding author. Fax: +81-11-706-7128

Email address: nao@athena.qe.eng.hokudai.ac.jp (Naoki Miyamoto).
} 
based on a thermal process as an optical phase modulation device. The nitroanisole exhibits a thermal lens effect[9-12], i.e. a temperature dependent refractive index, and it absorbs IR radiation as heat. Hence, the nitroanisole can induce phase modulation to visible light, in direct response to intensity of the incident IR radiation. The IR hologram, which are created by an IR reference wave and an IR object wave on the nitroanisole, function as a phase hologram for visible light. The proposed method can be used to obtain the IR hologram as the phase modulation distribution that corresponds to the IR intensity distribution on the nitroanisole by examining the phase map of visible light that is modulated upon passing through the nitroanisole. Once the IR hologram is extracted, we can reconstruct the intensity field and the phase map of the IR object wave by numerical calculation based on the Fresnel transform. In addition, IR holograms can be stored with sufficient detail since a high-resolution CCD that is sensitive to visible light is applied for recording the information of the IR holograms.

The aim of our work is to demonstrate experimentally the digital reconstruction of the IR holograms which are constructed on the nitroanisole. We first describe the procedure to obtain the phase modulation distribution, which function as a phase hologram for visible light, made by interference of IR beams on the nitroanisole. Then, we present the experimental results. The results show that both the amplitude and the phase of the IR wavefront can be reconstructed accurately. 


\section{$2 \quad$ Nitroanisole as an optical phase modulation device}

The nitroanisole has three chemical arrangements, namely $o_{-}^{-}, m_{-}^{-}$, and $p$ variants. We propose to use $o$-nitroanisole which is a colorless to slightly yellow liquid with a boiling point of $277^{\circ} \mathrm{C}$, a melting point of $9.4^{\circ} \mathrm{C}$, and molecular weight of 153.14 , as the optical phase modulation device. The nitroanisole has a large thermal lens effect, i.e. a strong temperature dependence on the refractive index. We tested to verify that the refractive index of the nitroanisole varied with temperature at a rate of $-1.1 \times 10^{-3}[1 / \mathrm{T}]$. In addition, we confirmed that the nitroanisole absorbs approximately $50 \%$ of incident IR laser irradiation of wavelength $10.6 \mu \mathrm{m}$ in case of that its thickness was $10 \mu \mathrm{m}$ and irradiation density was $2.0 \mathrm{~W} / \mathrm{cm}^{2}$. Therefore when the nitroanisole is irradiated by the IR laser, the temperature rises and the refractive index decreases. This effect causes phase modulation to visible light, in direct response to intensity of the incident IR laser on the nitroanisole. In case of that the IR holograms are created on the nitroanisole, the nitroanisole functions as a phase hologram for visible light. The IR holograms are created transiently while the nitroanisole is irradiated by the IR laser. Hence, the IR holograms will vanish instantly when the IR irradiation is stopped.

We established in previous research[13] that the nitroanisole has a spatial resolution of at least $8 \mathrm{lp} / \mathrm{mm}$, based on measurements of the diffraction efficiency dependent on the spatial frequency of the IR interference fringe. Therefore, it is expected that the nitroanisole has high spatial resolution compared with conventional IR cameras. Also, it would be easy to make a larger two-dimensional nitroanisole device that does not require a matrix structure, by filling the space between two substrates with nitroanisole. Thus, the proposed nitroanisole de- 
vice is much simpler and easy to handle compared with existing IR sensitive detectors that require a cryogenic system or matrix structure, etc.

In our method the converter material has to give the phase modulation to the probe laser with respect to the IR irradiation. In addition, the IR hologram have to be created transiently in order to obtain the IR hologram with utilizing the phase shifting method. Hence, the nitroanisole is one of the suitable material that satisfies these requirements.

\section{Extraction procedure of the IR holograms}

In this section, we describe the procedure for extraction of the phase modulation distribution, namely the IR hologram, that the nitroanisole imparts to incident visible light, in direct response to the irradiation of the IR beams. The optical setup under consideration is shown in Fig.1. It consists of two holographic recording setups. The first one is an off-axis holography setup in which the light source is an IR laser, and this one is used for creating an IR hologram, that subsequently may be reconstructed, on the nitroanisole. The other one is a digital holography setup based on a Mach-Zehnder interferometer in which the light source is a visible laser, and this one records the visible interference pattern modulated by passing through the nitroanisole on which the IR hologram is created, by utilizing the phase shifting method. By rotating a parallel plate, which was fabricated with accuracy of $\lambda / 10$, determinate phase shift required for use in the phase shifting method is given. We applied a general four step algorithm with $\pi / 2$ shift between each of four frames in order to derive an accurate phase map of the visible interference pattern registered by the CCD. 
Let us now consider the intensity distribution of the interferogram constructed by the IR object and referece wave at the nitroanisole. Supposing that the optical complex amplitude of the IR object wave and the IR reference plane wave on the nitroanisole plane $\xi-\eta$ are denoted respectively by $\boldsymbol{O}_{I R}(\xi, \eta)$ and $\boldsymbol{R}_{I R}(\xi, \eta)$, we can describe the intensity distribution of the IR hologram on the nitroanisole as

$$
I_{I R}(\xi, \eta)=\left|\boldsymbol{O}_{I R}(\xi, \eta)+\boldsymbol{R}_{I R}(\xi, \eta)\right|^{2}
$$

The IR object wave can be reconstructed by illuminating the amplitude hologram or the phase hologram corresponding to $I_{I R}(\xi, \eta)$ with reconstruction light or by numerical reconstruction of digitally stored hologram. In our study, numerical reconstruction of the IR wavefront is performed by means of extraction of the IR hologram from the visible interference patterns.

Next, we consider the intensity distribution of the visible interference pattern recorded by the CCD. Since the nitroanisole imparts to the incident visible laser only the phase modulation that corresponds to the IR intensity $I_{I R}(\xi, \eta)$, the wavefront just after transmission through the nitroanisole can be expressed as

$$
\begin{aligned}
\boldsymbol{O}_{v i s}(\xi, \eta) & =O_{v i s} \exp \left\{i \phi_{v i s}^{O}(\xi, \eta)\right\} \exp \left\{i \gamma I_{I R}(\xi, \eta)\right\} \\
& \equiv O_{v i s} \exp \left[i\left\{\phi_{v i s}^{O}(\xi, \eta)+\phi_{H}(\xi, \eta)\right\}\right]
\end{aligned}
$$

where $\gamma$ is a coefficient related to the degree of phase modulation, $O_{v i s}$ is the amplitude of the visible laser, $\phi_{v i s}^{O}(\xi, \eta)$ is the original phase map which is not related to the IR irradiation, $\phi_{H}(\xi, \eta)$ is corresponding to the phase modulation distribution of the phase hologram. Actually, the profile of the phase modulation distribution is not always identical to that of the IR intensity since 
the heat diffusion process is expected to occur in the nitroanisole. Here, the effect of this process is ignored in order to simplify the discussion. The wavefront $\boldsymbol{O}_{v i s}(\xi, \eta)$ is refocused keeping both phase and amplitude information on the CCD plane by means of double diffraction setup with lenses $L_{1}$ and $L_{2}$, hence the wavefront of $\boldsymbol{O}_{v i s}(x, y)$ on the CCD plane $x-y$ is represented by

$$
\boldsymbol{O}_{v i s}(x, y) \propto \boldsymbol{O}_{v i s}(\alpha \xi, \alpha \eta)
$$

where the magnification $\alpha$ is determined by the focal length of two lenses. Therefore, the phase modulation distribution $\phi_{H}(\xi, \eta)$ on the nitroanisole can be derived by examining the phase map of $\boldsymbol{O}_{v i s}(x, y)$. The visible reference wave is represented as

$$
\boldsymbol{R}_{v i s}\left(x, y ; \phi_{s}\right)=R_{v i s} \exp \left[i\left\{\left(\phi_{v i s}^{R}(x, y)+\phi_{s}\right)\right\}\right]
$$

where $\phi_{s}$ is the phase offset introduced by the phase stepping procedure. Hence the intensity distribution of the interference pattern, which is constructed by two visible wavefronts of $\boldsymbol{O}_{v i s}(x, y)$ and $\boldsymbol{R}_{v i s}(x, y)$ on the CCD, can be represented as

$$
\begin{aligned}
I_{v i s}\left(x, y ; \phi_{s}\right) & =\left|\boldsymbol{O}_{v i s}(x, y)+\boldsymbol{R}_{v i s}\left(x, y ; \phi_{s}\right)\right|^{2} \\
& =O_{v i s}^{2}+R_{v i s}^{2}+2 \cos \left\{\phi(x, y)+\phi_{s}\right\}
\end{aligned}
$$

where $\phi(x, y)$ is denoted by

$$
\begin{aligned}
\phi(x, y) & =\phi_{v i s}^{R}(x, y)-\phi_{v i s}^{O}(x, y) \\
& \equiv \phi_{v i s}(x, y),
\end{aligned}
$$

in the case without the IR hologram, or by

$$
\phi(x, y)=\phi_{v i s}(x, y)+\phi_{H}(x, y)
$$


in the case with the IR hologram. In the four step algorithm, by substituting registered intensities with stepped phase differences into the following equation, we can derive the phase map $\phi(x, y)$.

$$
\phi(x, y)=\tan \left\{\frac{I_{v i s}\left(x, y ; \frac{3}{2} \pi\right)-I_{v i s}\left(x, y ; \frac{1}{2} \pi\right)}{I_{v i s}(x, y ; 0)-I_{v i s}(x, y ; \pi)}\right\}^{-1} .
$$

In order to extract the IR hologram, the $\phi(x, y)$ must be obtained in the same way in both cases, i.e. with and without the IR hologram on the nitroanisole. The extracted phases calculated by Eq.(8) are wrapped into a range between $-\pi$ and $\pi$. Then, phase unwrapping is performed by comparing the phase difference between adjacent pixels in order to obtain the continuous phase maps. The phase modulation distribution of $\phi_{H}(x, y)$ can be extracted as difference between two unwrapped phase maps, with and without the IR hologram. Considering the magnification of the wavefront, we can derive the phase modulation distribution $\phi_{H}(\xi, \eta)$, i.e. IR hologram, by expansion or by reduction of the phase map $\phi_{H}(x, y)$.

In digital holography, the reconstructed wavefront $\psi(a, b)$, which is in the observation plane $a-b$ at a distance $d$ from the hologram plane $\xi-\eta$, can be obtained by calculating through the Fresnel-Kirchhoff integral, namely,

$$
\begin{aligned}
\psi(a, b)= & C \exp \left[i \frac{\pi}{\lambda d}\left(a^{2}+b^{2}\right)\right] \iint \boldsymbol{R}(\xi, \eta) \exp \left\{i \phi_{H}(\xi, \eta)\right\} \\
& \exp \left[i \frac{\pi}{\lambda d}\left(\xi^{2}+\eta^{2}\right)\right] \exp \left[-i \frac{2 \pi}{\lambda d}(a \xi+b \eta)\right] d \xi d \eta
\end{aligned}
$$

where $C$ is a complex constant, and $\boldsymbol{R}(\xi, \eta)$ is the numerical model of the reference wave. The term $\boldsymbol{R}(\xi, \eta) \exp \left\{i \phi_{H}(\xi, \eta)\right\}$ in Eq.(9) represents the wavefront obtained by illuminating the phase hologram in which the phase modulation distribution is $\phi_{H}(\xi, \eta)$ with reference wave $\boldsymbol{R}(\xi, \eta)$. The discrete form of 
Eq.(9) is derived using the pixel size of the CCD device, and to this form we apply the two-dimensional Fast Fourier Transform algorithm to the discrete samples. According to the reconstruction theory of holography, the reconstructed wavefront $\psi(a, b)$ must contain three components: zero-order diffracted light, the real image and the virtual image of the object. These three components of a digitally reconstructed image should appear separated completely since the IR hologram is created by an off-axis setup in our experiments. In the case where $\boldsymbol{R}(\xi, \eta)=\boldsymbol{R}_{I R}(\xi, \eta)$ and moreover $d$ is equivalent to the distance between the object and the nitroanisole, the IR object wave should be reconstructed with the correct amplitude and phase distribution corresponding to the original.

The procedure to reconstruct the IR wavefront by calculating the extracted phase hologram is summarized in Fig.2.

\section{Experiments and results}

The experimental setup is shown in Fig.1. A continuous wave (c.w.) $\mathrm{CO}_{2}$ laser operating in the fundamental Gaussian mode at a wavelength $10.6 \mu \mathrm{m}$ was used to construct the IR hologram on the nitroanisole and a He-Ne laser of 632.89nm wavelength was used as a light source in a Mach-Zehnder interferometer to record the interference pattern that includes the information of the IR hologram. The nitroanisole was filled between the glass substrate and ZnSe, which is an IR transmitting material. A film spacer was used to adjust the thickness between the two substrates to about $100 \mu \mathrm{m}$. Irradiation density of the $\mathrm{CO}_{2}$ laser on the nitroanisole cell was about $1.5 \mathrm{~W} / \mathrm{cm}^{2}$. In our experiment, the focal length of the lenses used in the double diffraction optical geometry 
were $f_{1}=15 \mathrm{~cm}$ and $f_{2}=20 \mathrm{~cm}$ respectively. The CCD camera with resolution of $3026 \times 2018$ pixels and size of $23.7 \times 15.6 \mathrm{~mm}^{2}$ was used for registering the visible interference patterns. The angle between the two IR beams produced an interference fringe of which the spatial frequency was about $6 \mathrm{lp} / \mathrm{mm}$.

\subsection{Extraction of the IR interference fringe}

First, we attempted to extract the simple interference fringe pattern constructed by the IR beams without using an aperture. Examples of the visible interference patterns captured by the CCD are shown in Fig.3(a),3(b). These images were recorded without IR interference fringe on the nitroanisole, and with the fringe, respectively. The small fringe shown in the center part of Fig.3(b) was caused by a phase change that was given by the passing through the nitroanisole on which the IR interference fringe, i.e. the periodical distribution of phase modulation, was constructed. Actually, the refractive index of the glass plate which forms a part of the nitroanisole cell may be also slightly changed, however the phase shift caused by the glass plate is negligible compared with the contribution of the nitroanisole. We conducted the phase-shifting procedure in each case, i.e. with and without the IR interference fringe, and phase unwrapping was performed in order to obtain continuous phase maps. Unwrapped phase maps of the center region are shown in Fig.4(a),4(b). Subsequently, by taking the difference (by subtraction) between the two phase maps of Fig.4(a) and Fig.4(b), we extracted the phase modulation distribution $\phi_{H}(x, y)$ that was related with IR interference pattern. The extraction result of the phase modulation and its one-dimensional profile are shown in Fig4(c), 4(d) respectively. A periodical distribution corresponding to 
the IR interference fringe pattern was observed. Because of thermal dispersion in the nitroanisole, the profile of the phase modulation distribution was not identical to that of the IR intensity distribution. However, we can estimate the IR fringe profile from the extracted phase modulation distribution since the phase modulation should be roughly equal to a superposition of periodical profile and fundamental profile that are corresponding respectively to the IR interference fringe and the $\mathrm{CO}_{2}$ laser mode.

The scale of each figure in Fig. 4 was $0.8 \mathrm{~mm} / 100$ pix. Therefore, the spatial frequency of the IR interference fringe, as derived by the extraction procedure from the recorded intensities obtained with the CCD, was about $4.5 \mathrm{lp} / \mathrm{mm}$. Based on the magnification of the wavefront, the actual spatial frequency of the IR interference fringe on the nitroanisole was estimated to about $6.0 \mathrm{lp} / \mathrm{mm}$. This spatial frequency was approximately identical to the actual value estimated from measurement by optical geometry.

In case of using the recording device, which has an elementary pixel size of $50 \mu \mathrm{m}$, to record the IR interference fringe in these experimental conditions, the sampling rate will be about $1 \mathrm{lp} / 4$ pix and this value is considered as the limit of phase-shifting digital holography. On the other hand, the proposed method can derive the IR interference fringe as the phase modulation with a sufficiently high sampling rate, and the nitroanisole device still has some margin to improve spatial resolution. Therefore, it is conceivable that the IR holograms can also be derived as phase modulation distribution with enough sampling number. 


\subsection{Digital reconstruction of the IR object wave}

Next, we attempted to reconstruct the IR wavefront that passed through the two-dimensional aperture by numerical calculations based on the extracted phase modulation distribution. The aperture, shaped like a letter "F" with lateral size and line width of about $5 \mathrm{~mm} \times 4 \mathrm{~mm}$ and $1 \mathrm{~mm}$, respectively, was used as the object to be reconstructed as shown in Fig.1. The distance $d$ from the aperture to the nitroanisole was $24 \mathrm{~cm}$. The IR hologram is created on the nitroanisole as a result of the interference of a plane reference wave and the object wave that is diffracted by passing through the aperture. The extracted phase modulation distribution $\phi_{H}(x, y)$, which was processed as described in the above section, is shown in Fig.5(a). Then, we conducted the numerical reconstruction of the wavefront $\psi(a, b)$ by calculating through Eq.(9). Here, the wavenumber of the reference wave $\boldsymbol{R}_{I R}(\xi, \eta)$ on the nitroanisole could not be obtained accurately since the incident angle between the $\boldsymbol{R}_{I R}(\xi, \eta)$ and $\boldsymbol{O}_{v i s}(\xi, \eta)$ varied slightly in our experiments. Therefore, we estimated the approximate wavenumber of $\boldsymbol{R}_{I R}(\xi, \eta)$ from the optical alignment and fringe distribution of the IR interference pattern as shown in Fig.4(c),4(d). The intensity field $|\psi(a, b)|^{2}$, that was calculated by substituting the distance $d$, the estimated wavenumber and the magnified phase modulation $\phi_{H}(\xi, \eta)$ into Eq.(9), is shown in Fig.5(b). The real image and the virtual image that was out of focus were respectively reconstructed in the left part and the right part in Fig.5(b). The real image of the letter "F" was reconstructed with the same size of the original aperture as shown in Fig.5(c). The reconstructed phase distribution $\operatorname{Arg}[\psi(a, b)]$ is shown in Fig.5(d). A constant phase value pattern corresponding to the letter " $F$ " can be observed in the phase map at 
the same position as the real image. As a result, it was confirmed that not only amplitude but also phase information concerning the IR object wave can be reconstructed.

A few causes are surmised as the reason why the reconstructed images contained undesired diffracted components and other noise. Firstly, it was possible that the state of the temperature distribution in the nitroanisole was slightly altered in each recorded frame of different phase-shifts because of the instability of the $\mathrm{CO}_{2}$ laser. By using the more stable $\mathrm{CO}_{2}$ laser, this affect can be eliminated. Additionally, it was surmised that the uncertainty of the optical setup, especially of the double diffraction setup, affected the phase map. Hence the two lenses have to be arranged accurately in consideration of their focus length.

\section{Conclusion}

An experimental method utilizing nitroanisole as an optical phase modulation device for the extraction of IR holograms was presented. Digital holography is one of the effective technique that can reconstruct not only amplitude but also phase of the object wave. However the spatial resolution of the conventional IR camera is not enough to record the IR hologram. Our proposed method can derive the IR hologram as the phase modulation with a sufficiently high sampling rate. It was confirmed that the amplitude and the phase information of the IR wavefront can be reconstructed numerically from the extracted phase modulation distribution. Hence, it is conceivable that the proposed method can be applied for phase measurements in the IR region, which may be useful for instance, in the analysis of deformation, optical flatness measurement, etc. 


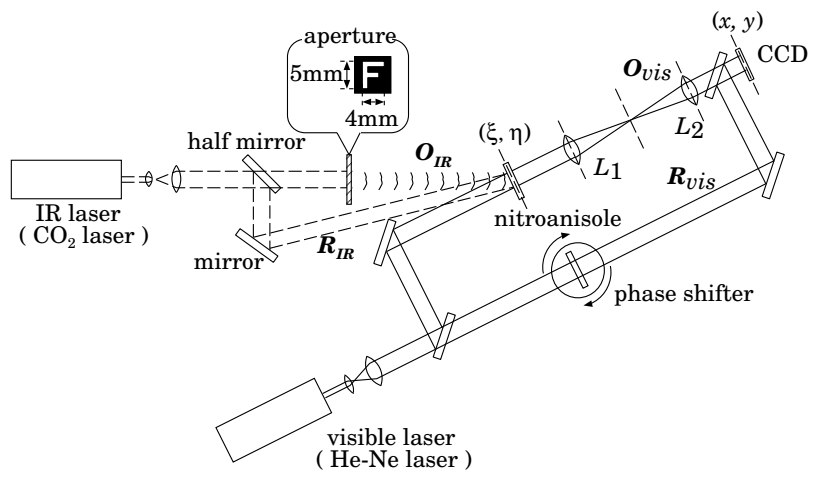

Fig. 1. The optical setup for the extraction of the IR hologram. 
without IR hologram with IR hologram

\begin{tabular}{l|l|l}
\hline registered intensities & $I_{0}, I_{1}, I_{2}, I_{3}$ & $I_{0}, I_{1}, I_{2}, I_{3}$ \\
$\begin{array}{l}\text { unwrapped phase map } \\
\text { on the CCD }\end{array}$ \\
$\begin{array}{c}\text { phase shifting \& phase unwrapping } \\
\text { on the nitroanisole }\end{array}$
\end{tabular}

Fig. 2. Procedure to reconstruct the IR wavefront. 


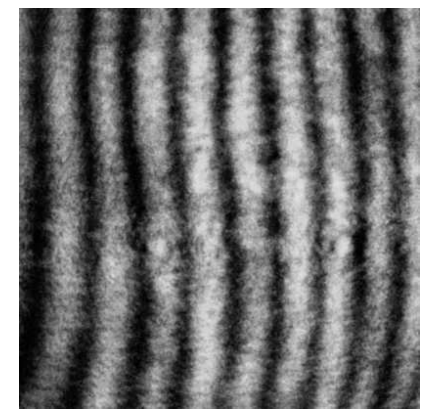

(a)

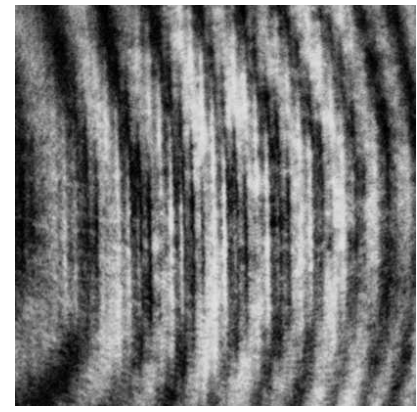

(b)

Fig. 3. Examples of interference patterns recorded by the CCD (a) without an IR interference fringe and (b) with an IR interference fringe. 


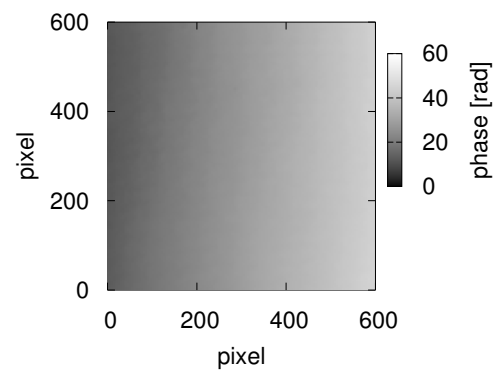

(a)

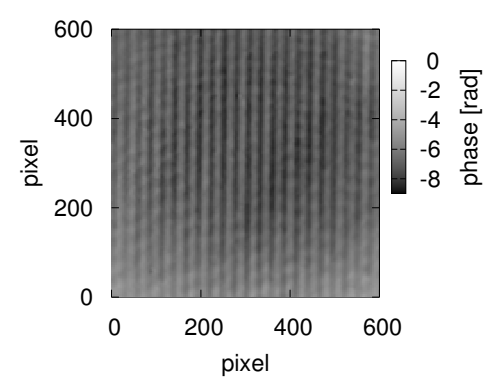

(c)

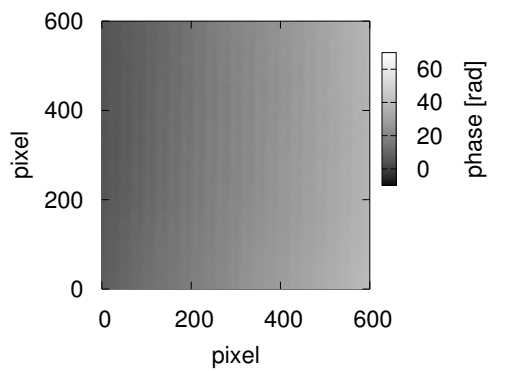

(b)

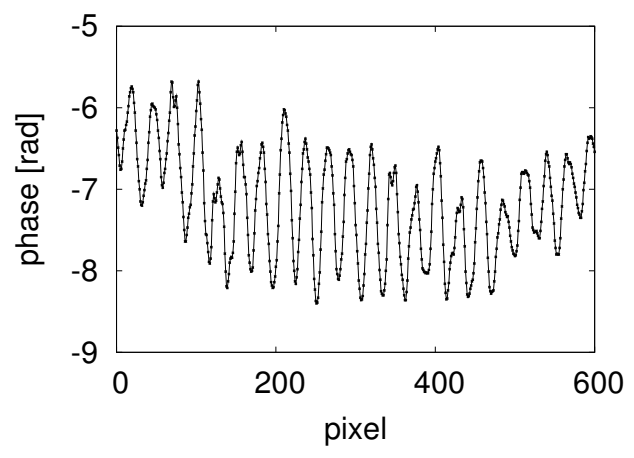

(d)

Fig. 4. Unwrapped phase maps; (a) without the IR hologram $\phi_{v i s}(x, y)$, (b) with the IR hologram $\phi_{H}(x, y)+\phi_{v i s}(x, y)$. Extracted phase modulation $(\mathrm{c}) \phi_{H}(x, y)$ and (d) its one-dimensional profile. 


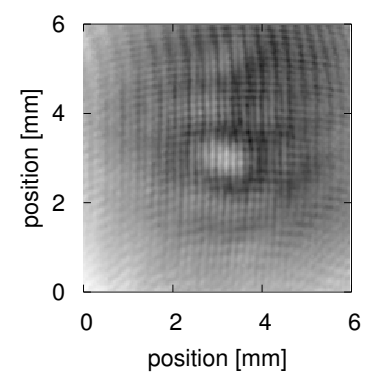

(a)

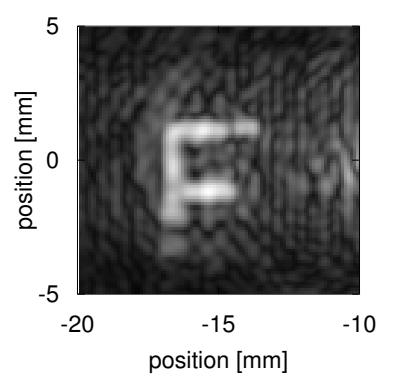

(c)

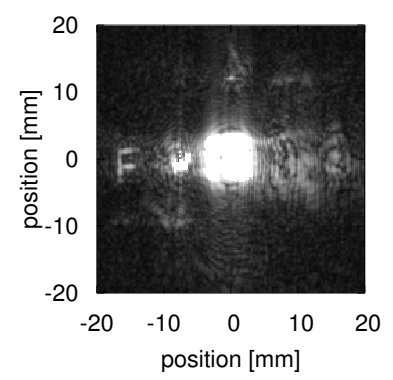

(b)

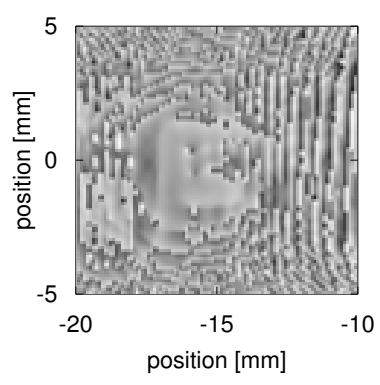

(d)

Fig. 5. (a) Extracted IR hologram $\phi_{H}(\xi, \eta)$. Reconstructed images; (b) intensity field $|\psi(a, b)|^{2}$ of real image(left side), virtual image(right side) and zero-order diffracted light, (c) intensity and (d) phase map $\operatorname{Arg}[\psi(a, b)]$ of the real image. 


\section{References}

[1] J. H. Bruning, D. R. Herriott, J. E. Gallagher, D. P. Rosenfeld, A. SD. White, D. J. Brangaccio, Appl. Opt. 13 (1974) 2693-2703.

[2] V. Srinivasan, H. C. Liu, M. Halioua, Appl. Opt. 23 (1984) 3105-3108.

[3] I. Yamaguchi, S. Ohta, J. Kato, Opt. Lasers Eng. 36 (2001) 417-428.

[4] E. Allaria, S. Brugioni, S. De Nicola, P. Ferraro, S. Grilli, R. Meucci, Opt. Commun. 215 (2003) 257-262

[5] S. Calixto, Appl. Opt. 27 1977-1983 (1988).

[6] J.M. Yang, D.W. Sweeney, Appl. Opt. 18 (1979) 2398-2406.

[7] F. Ledoyen, J. Lewandowski, M. Cormier, in Holographic Optics III: Principles and Applications, G. M. Morris, eds.,Proc. of SPIE 1507 (1991) 328-338.

[8] R. Beaulieu, R. A. Lessard, in Applications of Photonic Technology 4, R. A. Lessard and G. A. Lampropoulos, eds., Proc. of SPIE 4087 (2000) 1298-1301.

[9] J. P. Gordon, R. C. C. Leite, R. S. Moore, S. P. S. Porte, and J. R. Whinnery, Appl. Opt. 36 (1964) 3-8.

[10] Y. Kohanzadeh, Kwing Wai Ma, J. R. Whinnery, Appl. Opt. 12 (1973) 15841587.

[11] S. J. Sheldon, L. V. Knight, J. M. Thorne, Appl. Opt. 21 (1982) 1663-1669.

[12] F. Jürgensen and W. Schröer, Appl. Opt. 34 (1995) 41-50

[13] N. Miyamoto, S. Nisiyama, S. Tomioka, T. Enoto, Opt. Commun. 260 (2006) $25-29$. 